Felix Hirschfeld: Untersuchungen üb. den Eiweissbedarf des Menschen. 533

\title{
Untersuchungen über den Eiweissbedarf des Menschen.
}

Von

Felix Hirschfeld, pract. Arzt.

Im Anschluss an die Arbeiten des Pflüger'schen Laboratoriums, deren Ergebnissen nach der tägliche Eiweissumsatz bei dem Menschen etwas geringer zu sein schien, als man bisher angenommen hatte, forderte mich Herbst v. J. Herr Dr. Landwehr, der Leiter des Würzburger chemisch-physiologischen Instituts, auf, ebenfalls nach der Kjeldahl'schen Methode N-Bestimmungen des Urins vorzunehmen und stellte mir dazu die Mittel seines Instituts in der liebenswürdigsten Weise zur Verfügung.

Die Untersuchungen beschränkten sich damals darauf, die Menge der N-Ausscheidung bei den verschiedenen physiologischen Zuständen festzustellen. Durch ein unvorhergesehenes Ereigniss wurden die Arbeiten jedoch damals abgebrochen. Erst Juni l. J. konnte ich sie wieder aufnehmen und war jetzt der Zweck meiner Untersuchungen, die Grösse des Eiweissbedarfs für den Menschen zu bestimmen, d. h. inwieweit sich noch das Eiweiss durch andere Nahrungsstoffe vertreten lasse.

Ansgehend von zahlreichen Versuchen am Hund hatte $V$ o it dann die Ernährungsgesetze des Mensehen festgestellt. Er beobachtete, dass ein Versuchshund bei einem Gewicht von ca. $30 \mathrm{~kg}$ im Hunger constant ca. 6 gr N d. s. ca. $40 \mathrm{gr}$ Eiweiss oder $170 \mathrm{gr}$ Muskelfleisch zersetzte ${ }^{2}$ ). Trotzdem konnte derselbe bei Genuss von $500 \mathrm{gr}$ Fleisch nur dann sich auf dem N-Gleichgewicht erhalten, d. h. er zersetzte nicht mehr Fleisch, als ihm in der Nahrung geboten war, wenn ihm reichliche Mengen Fett oder Kohlehydrate gereicht wurden.

1) Pf 1 üg e r's Archiv, Band 36 und 38, Die Untersuchungen von Pflüger und Bohland und Bleibtreu und Bohland.

2) Voit, Handbuch des Stoffwechsels, S. 113. 
Diese Thatsache schien mit den bei dem Menschen beobachteten Verhältnissen in gutem Zusammenhang zu stehen. Bei längerem Hunger war bei Geisteskranken immer eine constante $\mathrm{N}$ Ausscheidung von ca. $4-6 \mathrm{gr}$ gefunden worden ${ }^{1}$ ), so auch neuerdings von Tuczek²). Vor kurzem konnte in der bekannten 10 tägigen Hungerperiode $\mathrm{Cettis}^{3}$ ) allerdings keine immer gleichbleibende Ausscheidung gefunden werden, vielmehr sank dieselbe ganz allmählich von 13 auf $10 \mathrm{gr} \mathrm{N}$. Munk bezog dies darauf, dass bei der Versuchsperson ein ausserordentlich eiweissreicher und fettarmer Organismus vorlag und die Harnstoffausscheidung noch durch reichliches Wassertrinken vermehrt war. Jedenfalls wurden anch vom Menschen im Hunger immer geringere Mengen $\mathrm{N}$ ausgeschieden, als dies nach den verschiedensten Beobachtern bei den meisten Bevölkerungsklassen am häufigsten der Fall war.

Im Wesentlichen nahm man an, müsste der in der Nahrung enthaltene $\mathrm{N}$ in Eiweiss geboten werden. Entsprechend den im Urin meist gefundenen Werthen von $15-20 \mathrm{gr} N$ glaubte man daher, dass bei normaler Resorption ca. 100-130 gr Eiweiss für den erwachsenen Mann nothwendig seien. Der Frage, in wie weit es möglich wäre, dass für einen Theil dieser Liweissmenge andere Nahrungsstoffe als Ersaty eintreten könne, war man bisher, wenn man von einigen älteren Versuchen absieht, nicht näher getreten. Nur am Thier angestellte Experimente lagen hierüber vor. Nach diesen erscheint als bestes Ersatzmittel für Eiweiss der Leim, wern man die Peptone nicht berücksichtigt, die wohl für gewöhnlich als Nahrungsmittel nicht in Betracht kommen.

Der Leim wird unter normalen Verhältnissen ${ }^{4}$ ) schon in der Nahrung als leimgebendes Gewebe in den Sehnen dem Bindegewebe des Fleisches in verschiedener Menge genossen. Er enthält ${ }^{5}$ ) procentuarisch weniger $\mathrm{C}, \mathrm{N}$ und $\mathrm{S}$ als das Eiweiss, es fehlt ihm wahrscheinlich die aromatische Gruppe.

Nach den Untersuchungen von $\mathrm{V}$ o it ist es möglich, durch reichliche Darreichung von Leim den Eiweissverlust des Körpers

1) Vo it, Handbuch des Stoffwechsels, S. 84.

2) Tu c z e k cit. nach $\mathrm{M}$ a ly, Jahresbericht XV, 401.

3) Berlin. Klin. Wochenschrift 1887, S. 428.

4) Vo it, Handbuch des Stoffwechsels, S. $395 \mathrm{u}$. f.

5) M unk und Uffel $\mathrm{mann}$, Handbuch der Dïttet. S. 36. 
oder den Bedarf des Körpers an eiweisshaltigen Bestandtheilen bedeutend herabzusetzen. Schliesslich hat nach Voit der Organismus nur so viel Eiweiss nothwendig, als er zum Ersatz der zu Grunde gegangenen Blutkörperchen und Epithelien bedarf. Der Leim wird dabei rasch und vollständig zerstört. Eine Bildung von Eiweiss aus Leim oder ein Zurückbleiben von ihm als solchen darf daher nicht angenommen werden, da er rasch nach seiner Aufnahme aus dem Körper auch entfernt wird, wenigstens sich seine N-haltigen Bestandtheile als Harnstoff auffinden lassen. Ebenso oder ähnlich verhalten sich die leimgebenden Gewebe, Sehnen u. s. w., welche noch besonders die Vorziuge haben, dass sie verhältnissmässig raseh verdaut und resorbirt werden.

$V$ o it ${ }^{1}$ ) sagt iiber diesen Gegenstand:

„Der Leim erspart Eiweiss in viel höherem Grade als das Fett und die Koblehydrate, denn 100 Theile Leim ersetzen 50 Theile Eiweiss und er wird in dieser Beziehung nur vom Pepton übertroffen." Und weiter :

„Es muss jedoch stets zu dem Leim eine gewisse Menge Eiweiss hinzugesetzt werden, um den Eiweissbestand des Körpers zu erhalten."

Nach diesen Resultaten wird es theilweise begreiflich, dass man eigentlich verzichtete, dem Mensehen Ersatzmittel für das Eiweiss zu reichen, da weder Pepton noch Leim gut in grösserer Menge genossen werden können. Dabei erscheint es nun wichtig zu wissen, warum gerade diese Stoffe so geeignet erscheinen und nicht die Fette oder Kohlehydrate, denn da der Leim doch nicht zum Aufbau des Organismus dient, könnte es nur die Zersetzung $\mathrm{N}$-haltiger Atomencomplexe sein, welche für den Organismus so wichtig wäre. Man musste demnach zwei Ursachen für den Eiweissbedarf annehmen.

Erstens den Wiederersatz des zu Grunde gegangenen organisirten Zellenmaterials, zweitens die Zersetzung einer gewissen Menge N-haltiger Bestandtheile, mögen dieselben in Eiweiss oder Leim gereicht werden. Der Unterschied zwischen dieser Annahme und der früheren sogenannten Luxustheorie wäre demnach nur der, dass nach dieser die Zersetzung dieses überschüssigen Eiweissmaterials als unniitz fiur den Organismus bezeichnet wurde, wäh-

1) Vo it, Handbuch des StoffwechseIs, S, 400. 
rend man jetzt diesen Vorgang der Spaltung und Ausscheidung des Eiweisses oder Leims als unentbehrlich für die Existenz der Zelle hinstellt.

Erkennt man diesen Satz nicht an, so mïsste man den schon unter normalen Verhältnissen in grosser Menge genossenen Nahrungsstoffen, den Fetten und Kohlehydraten, die gleiche Rolle in Bezug auf den Eiweissersatz zuerkennen. Oder man muisste annehmen, dass bei der Leimnahrung die Eiweisszersetzung im Organismus, beziehungsweise der Eiweissverbrauch sich nach ganz anderen Normen regelt, als wenn nur Eiweiss, Fette und Koblehydrate ohne Leim oder nur mit so viel, als in dem hauptsächlichsten eiweisshaltigen Nahrungsmittel dem Fleisch enthalten ist, genossen werden.

Die Kohlehydrate scheinen jedoch nach den meisten Untersuchungen keinen so günstigen Einfluss als eiweisssparende Nahrungsmittel auf den Organismus zu entfalten. Sie können nach Vo it ${ }^{1}$ ) den Eiweissumsatz nur um durchschnittlich $9 \%$, im höchsten Falle $15 \%$ herabsetzen. Auch den Fetten ${ }^{2}$ ) misst er in dieser Beziehung keine höhere Bedeutung bei. Neuerdings untersuchte Rubner diese Verhältnisse. Er konnte beim Hunde die N-Ausscheidung im Hunger dureh reichlichen Genuss von Kohlehydraten besonders Zucker in $4-5$ Tagen um $30-40 \%$ herabsetzen ${ }^{3}$ ). Beim Huhn gelang ihm dies aus ihm unbekannten Gründen allerdings nicht in dem Grade.

„Für einen idealen Zustand, so führt er ${ }^{4}$ ) aus, wäre das Eiweiss nur für die Wachsthumsvorgänge (im weitesten Sinne) im Organismus nöthig; wenn nun dasselbe unter den angegebenen Bedingungen auch zum Theil zerfällt, so ist doch dieser Zerfall zu unbedeutend, um irgend für den Kraftwechsel in Betracht zu kommen, für welchen letzteren alle Stoffe die Quelle darstellen können."

Schliesslich fügt er jedoch hinzu:

"Ich hebe jedoch nochmals hervor, dass man aus dem geringen Verbrauch eiweissartigen Materials bei Zufuhr $\mathrm{N}$-freier

1) Voit, Handbuch des Stoffwechsels, S. 140.

2) Voit, ebenda, S. 127.

3) $R$ u b n e r, Die Vertretungswerthe der hauptsächlichsten organischen Nahrungsstoffe im Thierkörper, Zeitschr. f. Biol. XIX.

4) Ebenda, S. 393. 
Stoffe nicht auf eine untergeordnete Bedeutung des ersteren schliessen darf, wenn die Anfgabe gestellt ist, den N-Bestand eines Organismus zu erhalten. Wie schon früher einmal gesagt wurde, kann man das Letztere nur dann erreichen, wenn die mehrfache Menge des bei Hunger oder N-freier Kost zerstörten Eiweisses zugeführt wird."

Von noch grösserem Werth ist eine Untersuchungsreihe von E. Salkowski ${ }^{1}$ ). Derselbe prüfte den Einfluss von Ammonsalzen auf die Harnstoffausscheidung. Das Thier bekam ea. 30 Tage lang immer dasselbe Futter, das ans ca. $50 \mathrm{gr}$ condensirter Milch, $50 \mathrm{gr}$ Speck und $150 \mathrm{gr}$ Brod bestand. Es ist hier also vermieden, dem Thier nur einen Nahrungsstoff zu bieten. Dadurch ist einestheils wohl eine bessere Resorption erzielt worden, andererseits wurde es möglich, das Thier so lange bei dieser Kost zu erhalten. Während dieser Zeit sank die $\mathrm{N}$-Ausscheidung im Urin auf 2,6 bis $3,0 \mathrm{gr}$. Rechnet man, dass ein ca. $30 \mathrm{~kg}$ schwerer Hund im Hunger, wie Voit constatirte, ca. 6gr $\mathrm{N}$ ausscheidet, so hätte bei obigem Versuchsthier bei einem Gewicht von $22 \mathrm{~kg}$ eine Tagesausscheidung von ca. $4,5 \mathrm{gr}$ erfolgen müssen, oder vielleicht eine noch grössere Menge, da kleinere Thiere einen verhältnissmässig regreren Eiweissumsatz haben.

Zieht man den N-Gehalt der Nahrung in Betracht²), so ist

$$
\begin{aligned}
50 \mathrm{gr} \text { condensirte Milch } & =6 \mathrm{gr} \text { Eiweiss } \\
150, \text { Brod } & =12,7 ", " \\
50, \text { Speck } & =1,5, ", \\
\cline { 2 - 3 } &
\end{aligned}
$$

d. s. $3,2 \mathrm{gr} \mathrm{N}$.

Es ist also wahrscheinlich, dass bei dieser ungenügenden Ernälrung, besonders da noch viel durch mangelnde Resorption in Verlust ging, doch das Thier täglich nur ca. $1 / 2 \mathrm{gr} \mathrm{N}$ d. s. $3 \mathrm{gr}$ Eiweiss von seinem Körper zusetzte!

Bei all diesen Versuchen ist beachtenswerth, dass als Versuchsthier der Hund, also ein wesentlich fleischfressendes Thier,

1) Zeitschr. für phys. Chemie, Band I, S. 43 u. 44.

2) Genaue Angaben iiber die Zusammensetzung der gebrauchten Nahrungsmittel konnte ich nicht erhalten, die Zahlen sind aus den später erwähnten Abhandlungen Voits und $\mathrm{Kön}$ ig entnommen. 
benutzt wurde. Dass für letzteres das vegetabilische Futter, als meist aus Kohlehydraten bestehend, ungeeignet erscheint, liegt auf der Hand. E. Bis $\mathrm{ch} \mathrm{off}^{1}$ ) sieht den Grund hierfür theilweise in der Kürze des Darms und darin, dass diese Nahrungsmittel, in seinem Versuche das Brod, grosse Neigung zu saneren Gährungen und Zersetzungen hatten.

Diesen Thatsachen gegentiber schien daher eine Untersuchung angezeigt, ob der Mensch nicht im Stande wäre, mit weniger Eiweiss, als bisher für ihn als nothwendig angenommen wurde, das $\mathrm{N}$-Gleichgewicht am Körper herzustellen, wofern er sich nur reichlich Kohlehydrate und Fette zuführte.

Schon Beneke ${ }^{2}$ ) wies nach, dass ein erwachsener Mann auch mit weniger als $100 \mathrm{gr}$ Eiweiss für den Tag sich erhalten könne. Ihm genügten bei allerdings leichter Arbeit in einer 15 tägigen Versuchsreihe durehschnittlich ca. $90 \mathrm{gr}$.

Auch Flügge beobachtete, dass ein Mann meist nur 8-10 gr $\mathrm{N}$ d. s. (entsprechend $50-62,5 \mathrm{gr}$ Eiweiss) in 24 Stunden im Urin ausschied. Doch betont er selbst, dass derselbe eine schwächliche Körperconstitution und geringe körperliche Leistungsfäbigkeit darbot.

Versuchsperson ist in unseren Untersuchungen Verfasser selbst. Derselbe ist 24 Jahre alt, 3,73 gross und bei gewöhnlich leichter Kleidung $73 \mathrm{~kg}$ schwer. Geringer Pannicul. adipos., ziemlich kräftige Muskulatur. Verf. war vor kurzem Soldat und glaubt daher, die Körperkräfte zu besitzen, welche $\operatorname{man}_{\star}^{\natural}$ bei einem Arbeiter, der mittelschwere Arbeit zu leisten hat, voraussetzt.

Die gewohnte tägliche Nahrung enthielt, wie im November 1886 vorgenommene Bestimmungen ergaben, ca. 100-130 gr Eiweiss. Die N-Ausseheidung im Urin schwankte zwischen 16 bis 20 gr N.

Als Nahrungsmittel wurden vorzüglich die an Kohlehydraten reichen und an Albuminaten armen Stoffe, Reis, Kartoffeln, Bier u. s. w. gewählt, von Fetten besonders Butter, in zweiter Linie Speck. Die Zubereitung war die denkbar einfachste, sowohl der äusseren Umstände halber als der genauen Controlle wegen.

1) E. B is ch off, Zeitschr.f. Biolog. V, S. 455.

2) Beneke, Zur Ernährungslehre des gesundeu Menschen.

3) Flüg g e, Beiträge zur Hygiene, S. 102. 
Die Kartoffeln wurden immer roh gewogen und in den Schalen gekocht. Die Abfallstoffe ergaben sich darauf als ca. $5 \%$ bis $7 \%$. Es wurden daher immer $530 \mathrm{gr}$ abgewogen, um $500 \mathrm{gr} \mathrm{zu}$ erhalten. Der N-Gehalt schwankte nach vom Verfasser vorgenommenen Analysen zwischen $0,32-0,35 \%$. Der Gehalt an N-Substanzen wäre demgemäss ca. 2,0-2,2\%. Letztere Zahl wurde angenommen, da sie mit der von $\mathrm{K} o ̈ n i{ }^{1}{ }^{1}$ ) für weissfleischige Kartoffeln als Mittelzahl gegebenen ubereinstimmt. Die Koblehydrate nabmen wir nach derselben Angabe als 20\% an. Der Reis ${ }^{2}$ ) wurde als 7\% Eiweissstoffe und 78\% Kohlehydrate haltend, das Brod als Semmel ${ }^{3}$ ) mit $60 \%$ Kohlehydraten und $10 \%$ eiweisshaltigen Stoffen gerechnet. Der Reis wurde meist in Form eines festen Breies mit reichlich Butter oder etwas Zucker verzehrt. Das Bier ${ }^{4}$ ) wurde als Sommerbier mit 0,5, als Exportbier mit 0,7\% Gehalt an N-haltigen Stoffen mit 6\% Extract- und $3 \%$ beziehungsweise $4 \%$ Alcoholgebalt gerechnet. Der Kaffee ${ }^{5}$ ), der täglich in einer Menge von ca. $20 \mathrm{gr}$ verwendet wurde, ist mit $3 \%$ $\mathrm{N}$-haltigen Stoffen (vorzüglich Caffein), der Wein $^{6}$ ) mit $0,2 \%$ Eiweissstoffen, $8 \%$ Alcohol und 1\% Zucker gerechnet. Der Butter legte man nach $\mathrm{V}_{0} \mathrm{it}^{7}$ ) eine Zusammensetzung von $0,9 \%$ Eiweissstoffen und 92\% Fett bei der Berechnung zu Grunde, dem Speck nach eigener Analyse ca. 3\% Eiweiss und 75\% Fett ${ }^{8}$ ).

Während der Versuchszeit wurden täglich durchschnittlich 2 Liter Bier und bisweilen etwas Wein getrunken. Wenn es Bedenken erregen könnte, dass also verhältnissmässig grosse Mengen Alcohol genossen wurden und dass dieser einen Einfluss auf den Stoffwechsel ausïben konnte, so ist zu erwägen, dass in hiesiger Gegend (in Bayern) von dem grössten Theil der Bevölkerung ähnliche Quantitäten meist getrunken werden und dass sie in dieser

1) J. K ö n i g, Chemische Zusammensetzung der menschl. Nahrungs- u. Genussmittel, Berl. 1879, S. 96.

2) J. Kön i g, Die menschl. Nahrungs- und Genussmittel, Berl. 1880, S. 285 .

3) Vo it, Handbuch des Stoffwechsels.

4) J. König, Die menschl. Nahrungs- und Genussmittel, S. 424.

5) J. Kö n i g, ebenda, S. 481.

6) J. Kön i g, ebenda, S. 457.

7) V o it, Handbuch des Stoffwechsels, S. 457.

8) J. Kö n ig, ebenda, S. 170. 
Form wohl als Nahrungsmittel gelten dürfen. Auch die Angabe, dass Alcohol den N-Umsatz verringere, verliert dadurch an Bedeutung, dass, wie die erwähnten Untersuchungen Rubner's zeigen, dies andere Nahrungsstoffe noch in viel höherem Maasse thun.

Die Verdauung und Resorption der Nahrung schien eine sehr günstige zu sein. Verdauungsbeschwerden traten nur einmal auf und verschwanden auf Genuss von etwas Rothwein rasch. Die Fäces wurden alle 2-3 Tage entleert, sie waren von geringer Menge, meist wasserarm und dunkelbraun. Sie wurden einmal untersucht und bestand, wenn man den N-Gehalt des Urins und der Fäces zusammenaddirte, doch kein Ueberschuss der N-Abgabe über die N-Aufnahme.

Der Urin war von derselben Färbung wie bei der gewöhnlichen eiweissreichen Ernährung. Er wurde immer frei von Eiweiss und trotz oft reichlichen Genusses von Zucker auch zuckerfrei befunden. Sein N-Gehalt schien von der Fluissigkeitsmenge nicht abhängig zu sein, da an den Tagen, an welchen mehr getrunken und auch demgemäss mehr Wasser ausgeschieden wurde, keine deutlich wahrnehmbare Steigerung der N-Ausfuhr eintrat.

Während beider Versuchsreiben, besonders in der ersten, wurde eine. grössere körperliche Arbeit geleistet, als Verfasser sonst gewohnt war. Täglich wurde eine Stunde - zu verschiedenen Tageszeiten in kleinen Perioden - mit Hanteln von ca. $12 \mathrm{~kg}$ kräftig gehantelt, 2-3 Stunden wurde ziemlich rasch spaziren gegangen, ausserdem täglich Bergbesteigungen von $2-300 \mathrm{~m}$ gemacht. Im iibrigen wurde im Verlauf des Tages ca. 6-7 Stunden Laboratoriumsarbeit verrichtet. In der zweiten Versuchsreihe legte Verfasser am 8 . beziehungsweise 9 . Versuchstage in 8 Stunden von früh $3 \mathrm{Uhr}$ bis $11 \mathrm{Uhr}$ einen Marsch von iuber $43 \mathrm{~km}$ in leicht bergiger Gegend zurück. Die körperliche Leistungsfähigkeit war durch diese Kost also durchaus nicht herabgesetzt. Vielmehr liess sich besonders an den Armen eher eine Vermehrung derselben erkennen.

Um dabei nicht durch den Sehweiss nicht genau zu controllirende Verluste zu erleiden, wurde an einzelnen Tagen möglichst wenig gearbeitet, so dass die Schweisssecretion dann fast vollständig unterdrïckt war. Hierbei trat nun keine wahrnehmbare Aenderung der N-Ausfuhr ein. Auch waren bei einem im Winter gemachten Versuch, wo nach 3 tägiger $\mathrm{N}$-armer Kost ein ca. 6 Stunden 
dauernder Marsch mit Bergbesteigungen gemacht wurde, ebenfalls geringe N-Mengen im Urin, während die Schweissabsonderung an diesem Tage kaum ins Gewicht fiel. Wenn man den geringen $\mathrm{N}$-Gehalt der Körpersäfte d. h. die geringe Menge von circulirendem Eiweiss in Betracht zieht, scheint es sehr wahrscheinlich, dass der iberdies nicht bedeutende $\mathrm{N}$-Gehalt des Schweisses hier noch niedriger war.

Das Gewicht sank in der ersten Versuchsreihe um ea. $1 / 2 \mathrm{~kg}$, in der zweiten Reihe liess sich eine Abnahme nicht constatiren.

I. Versuchsreihe.

15.-16. Juni.

$\begin{array}{lrccc} & \text { N-haltige Stoffe } & \text { Fette } & \text { C-Hydrate } & \text { Alcohol } \\ 500 \mathrm{gr} \text { Kartoffeln } & 11,0 & & 100 & \\ 150 \text { " Butter } & 1,4 & 138 & 156 & \\ 200 " \text { Reis } & 14,0 & & & \\ 100 " \text { Milch } & 4,0 & 4 & 4,0 & \\ 20 \text { " Caffee } & 0,6 & & & \\ 2 \text { Liter Bier } & 10,0 & & 120 & 60 \\ 1 / 2 \text { " Wein } & 1,0 & & 5,0 & 40 \\ 70 \mathrm{gr} \text { Zucker } & & & 70 & \\ & 42,0 \mathrm{gr} & 142 \mathrm{gr} & 455 \mathrm{gr} & 100 \mathrm{gr} .\end{array}$

Urin $980 \mathrm{ccm}$.

$10 \mathrm{ccm}$ entsprechen $7,0 \mathrm{ccm}$ Normalschwefelsäure $=0,098 \mathrm{gr} \mathrm{N}$.

Gesammtmenge des $\mathrm{N}$ im Urin $\quad 9,604 \mathrm{gr}$.

" " in der Nahrung 6,72 "

16.-17. Juni.

$\begin{array}{lcccc} & \text { N-haltige Stoffe } & \text { Fette } & \text { C-Hydrate } & \text { Alcohol } \\ 500 \text { gr Kartoffeln } & 11,0 & & 100 & \\ 150 " \text { Butter } & 1,4 & 138 & & \\ 150 " \text { Reis } & 10,5 & & 117 & \\ 40 " \text { Speck } & 1,2 & 30,0 & & \\ 100 \text { " Milch } & 4,0 & 4,0 & 4,0 & \\ 2 \text { Liter Bier } & 10,0 & & 120 & 60,0 \\ 70 \text { gr Zucker } & & & 70,0 & \\ 1 \text { Ei } & 6,0 & 5,0 & & \\ 20 \text { gr Caffee } & 0,6 & & & \\ & 44,7 \mathrm{gr} & 177 \mathrm{gr} & 411 \mathrm{gr} & 60 \mathrm{gr} .\end{array}$

Urin $970 \mathrm{ccm}$. 
$10 \mathrm{ccm}$ entsprechen $5,5 \mathrm{ccm}$ Normalschwefelsäure $=0,077 \mathrm{gr} \mathrm{N}$.

Gesammtmenge des $\mathrm{N}$ im Urin $\quad 7,469 \mathrm{gr}$.

" $\quad$ in der Nahrung 7,15 ,

17.-18. Juni.

$\begin{array}{lrccc} & \text { N-haltige Stoffe } & \text { Fette } & \text { C-Hydrate } & \text { Alcohol } \\ 500 \mathrm{gr} \text { Kartoffeln } & 11,0 & & 100 & \\ 140 " \text { Butter } & 1,3 & 129 & & \\ 150 " \text { Reis } & 10,5 & & 117 & \\ 30 " \text { Speck } & 0,9 & 22 & & \\ 1 \mathrm{Ei} & 6,0 & 5,0 & & \\ 100 \mathrm{gr} \text { Milch } & 4,0 & 4,0 & 4,0 & \\ 20 \text { "Caffee } & 0,6 & & & \\ 2 \text { Liter Bier } & 10,0 & & 120 & 60 \\ 1 / 2 \text { " Wein } & 1,0 & & 5 & 40 \\ 60 \mathrm{gr} \text { Zucker } & & & 60 & \\ & 45,3 \mathrm{gr} & 160 \mathrm{gr} & 406 \mathrm{gr} & 100 \mathrm{gr} .\end{array}$

Urin $1270 \mathrm{ccm}$.

$10 \mathrm{ccm}$ entsprechen $3,8 \mathrm{ccm}$ Normalschwefelsäure $=0,0532 \mathrm{gr} \mathrm{N}$.

Gesammatmenge des $\mathrm{N}$ im Urin $\quad 6,756 \mathrm{gr}$.

" in der Nahrung 7,25 ,

18.-19. Juni.

N-haltige Stoffe Fette C-Hydrate Alcohol

$500 \mathrm{gr}$ Kartoffeln

11,0

100

150 , Butter

1,4

10,5

138

150 , Reis

6,0

$1 \mathrm{Ei}$

4,0

$11 / 2$ Liter Bier

7,5

4,0

$1 / 4 \quad$ Wein

0,5

90,0

45

$20 \mathrm{gr}$ Caffee

0,6

2,0

20

60 "Zucker

$-41,5 \mathrm{gr} \quad \frac{60,0}{373 \mathrm{gr}} \frac{65 \mathrm{gr} .}{37 \mathrm{gr}}$

Urin $1160 \mathrm{ccm}$.

$10 \mathrm{ccm}$ entsprechen $3,4 \mathrm{ccm}$ Normalschwefelsäure $=0,0476 \mathrm{gr} \mathrm{N}$.

Gesammtmenge des $\mathrm{N}$ im Urin $\quad 5,52 \mathrm{gr}$.

$" \quad, \quad$ in der Nahrung 6,64, 


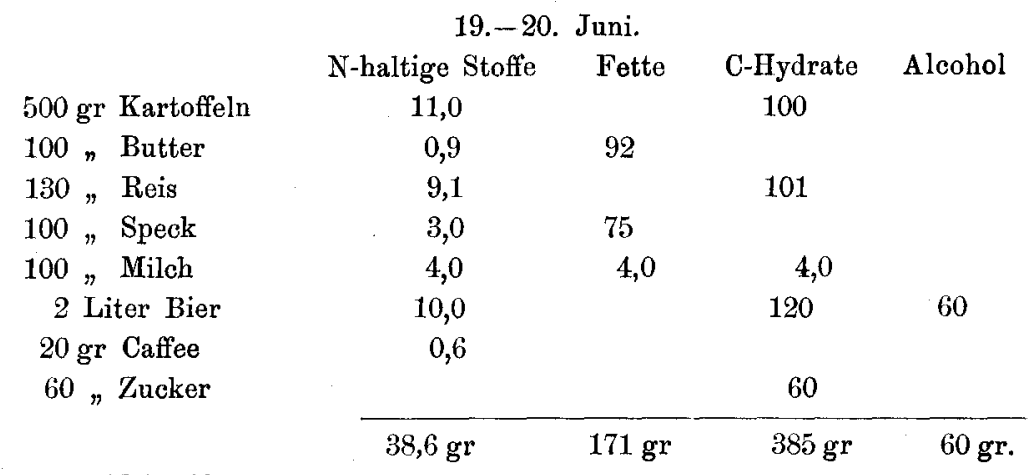

Urin $1200 \mathrm{ccm}$.

$10 \mathrm{ccm}$ entsprechen $3,4 \mathrm{ccm}$ Normalschwefelsäure $=0,0476 \mathrm{gr} \mathrm{N}$.

Gesammtmenge des $\mathrm{N}$ im Urin $\quad 5,71 \mathrm{gr}$.

" " in der Nahrung 6,18,

20.-21. Juni.

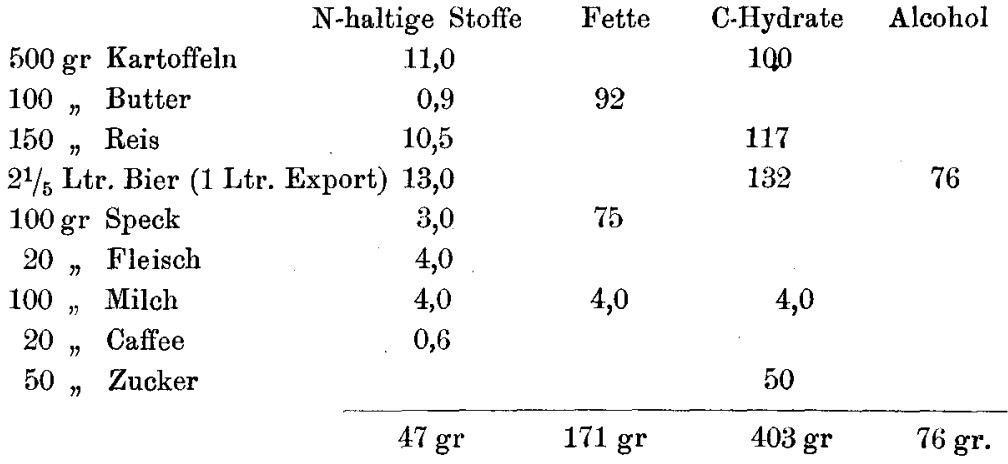

Urin $1520 \mathrm{ccm}$.

$10 \mathrm{ccm}$ entsprechen $2,5 \mathrm{ccm}$ Normalschwefelsäure $=0,035 \mathrm{gr} \mathrm{N}$.

Gesammtmenge des $\mathrm{N}$ im Urin $5,32 \mathrm{gr}$.

" , in der Nahrung 7,52,

21.-22. Juni.

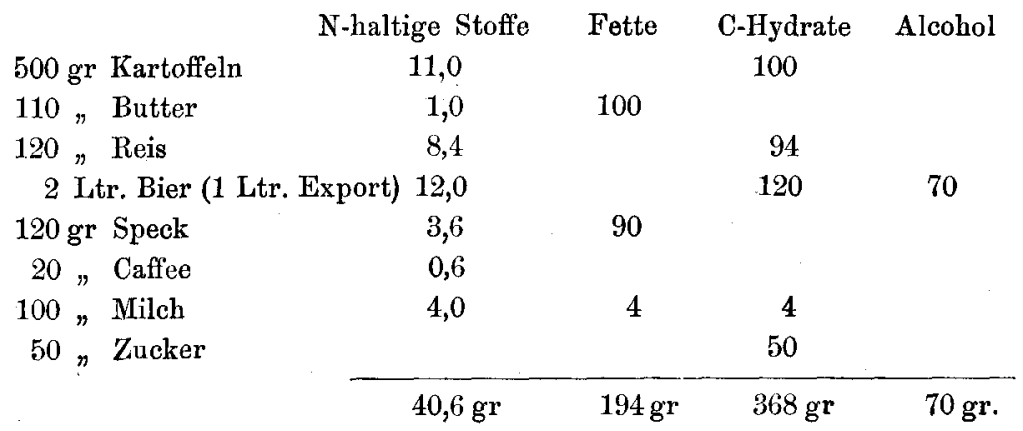


Urin $1480 \mathrm{ccm}$.

$10 \mathrm{~cm}$ entsprechen $2,6 \mathrm{ccm}$ Normalschwefelsäure $=0,0364 \mathrm{gr} \mathrm{N}$. Gesammtmenge des $\mathrm{N}$ im Urin $\quad 5,387 \mathrm{gr}$. $\Rightarrow \quad n$ in der Nahrung 6,3. . 22.-23. Juni.

\begin{tabular}{|c|c|c|c|c|}
\hline & N-haltige Stoffe & Fette & C.Hydrate & Alcohol \\
\hline $500 \mathrm{gr}$ Kartoffeln & 11,0 & & 100 & \\
\hline 120, Butter & 1,1 & 110 & & \\
\hline $1 / 2$ Liter Bier & 2,5 & & 30 & 15 \\
\hline $3 / 5 \quad, \quad$ Rothwein & 1,2 & & 6,0 & 48 \\
\hline $130 \mathrm{gr}$ Reis & $9, \mathbf{1}$ & & 101 & \\
\hline 20 , Caffee & 0,6 & & & \\
\hline 50, Milch & 2,0 & 2,0 & 2,0 & \\
\hline 40 , Zucker & & & 40 & \\
\hline & $27,5 \mathrm{gr}$ & $112 \mathrm{gr}$ & $279 \mathrm{gr}$ & $63 \mathrm{gr}$. \\
\hline
\end{tabular}

Urin $1250 \mathrm{ccm}$.

$10 \mathrm{ccm}$ entsprechen $2,8 \mathrm{~cm}$ Normalschwefelsäure $=0,0392 \mathrm{gr} \mathrm{N}$.

Gesammtmenge des $\mathrm{N}$ im Urin $4,9 \mathrm{gr}$.

" in der Nahrung 4,4,

23.-24. Juni.

N-haltige Stoffe Fette C-Hydrate Alcohol

$500 \mathrm{gr}$ Kartoffeln

180 , Butter

$1 / 2$ Liter Bier

50 gr Milch

150 , Reis

1 Liter Rothwein

$30 \mathrm{gr}$ Brod

50 "Speck

20 , Caffee

40 , Zucker
11,0

1,7

2,5

2,0

10,5

2,0

3,0

1,5

0,6
100

165

30

15

$2 \quad 2$

117

$10 \quad 80$

18

37

$\begin{array}{llll}34,8 \mathrm{gr} & 204 \mathrm{gr} & 317 \mathrm{gr} & 95 \mathrm{gr} .\end{array}$

Urin $1120 \mathrm{ccm}$.

$10 \mathrm{ccm}$ entsprechen $2,5 \mathrm{ccm}$ Normalschwefelsäure $=0,035 \mathrm{gr} \mathrm{N}$.

Gesammtmenge des $\mathrm{N}$ im Urin $\quad 3,92 \mathrm{gr}$.

$n \quad "$ in der Nahrung 5,56 , 


\begin{tabular}{lcccc} 
& \multicolumn{2}{c}{$24 .-25$. Juni. } & & \\
$500 \mathrm{gr}$ Kartoffeln & N-haltige Stoffe & Fette & C-Hydrate & Alcohol \\
150 " Butter & 11,0 & & 100 & \\
$11 / 2$ Liter Wein & 1,4 & 138 & & \\
$30 \mathrm{gr}$ Reis & 3,0 & & 15 & 120 \\
$2 \mathrm{gier}$ & 2,1 & & 23 & \\
$40 \mathrm{gr}$ Brod & 12,0 & 10 & & \\
50 "Zucker & 4,0 & & 24 & \\
$20 "$ Caffee & 0,6 & & 50 & \\
& $34,1 \mathrm{gr}$ & $148 \mathrm{gr}$ & $212 \mathrm{gr}$ & $120 \mathrm{gr}$.
\end{tabular}

Urin $1750 \mathrm{ccm}$.

$10 \mathrm{~cm}$ entsprechen $1,8 \mathrm{~cm}$ Normalschwefelsäure $=0,0252 \mathrm{gr}$ N.
Gesammtmenge des $\mathrm{N}$ im Urin $\quad 4,41 \mathrm{gr}$
" $"$ in der Nahrung 5,44 " 25.-26. Juni.

\begin{tabular}{|c|c|c|c|c|}
\hline $500 \mathrm{gr}$ Kartoffeln & 11,0 & 然 & 100 & 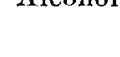 \\
\hline 160, Butter & 1,5 & 147 & & \\
\hline 100, Reis & 7,0 & & 78 & \\
\hline 60, Milch & 2,4 & 2 & 2 & \\
\hline 30, Brod & 3,0 & & 18 & \\
\hline 30 , Caffee & 0,9 & & & \\
\hline $60 "$ Speck & 1,8 & 45 & & \\
\hline $1 \mathrm{Ei}$ & 6,0 & 5 & & \\
\hline 1 Liter Bier & 5,0 & & 60 & 30 \\
\hline \multirow[t]{2}{*}{$30 \mathrm{gr}$ Zucker } & & & 50 & \\
\hline & $38,6 \mathrm{gr}$ & $199 \mathrm{gr}$ & $308 \mathrm{gr}$ & $30 \mathrm{gr}$ \\
\hline
\end{tabular}

Urin $900 \mathrm{~cm}$.

$10 \mathrm{ccm}$ entsprechen $4,8 \mathrm{ccm}$ Normalschwefelsäure $=0,0672 \mathrm{gr} \mathrm{N}$. Gesammtmenge des $\mathrm{N}$ im Urin $\quad 6,048 \mathrm{gr}$.

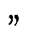

$$
\begin{aligned}
& \text { " in der Nahrung 6,2 " } \\
& \text { 26.-27. Juni. }
\end{aligned}
$$

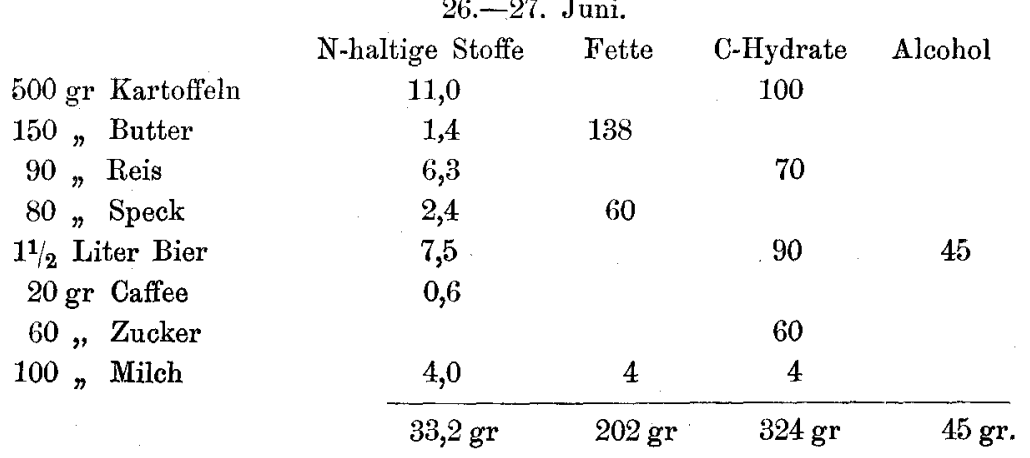

Urin $890 \mathrm{ccm}$.

$10 \mathrm{ccm}$ entsprechen $4,0 \mathrm{~cm}$ Normalschwefelsäure $=0,056 \mathrm{gr} \mathrm{N}$. 
Gesammtmenge des $\mathrm{N}$ im Urin

$4,984 \mathrm{gr}$.

$n$ $"$ " in der Nabrung 5,3 " 27.-28. Juni.

\begin{tabular}{|c|c|c|c|c|}
\hline & $\mathrm{N}$-haltige Stoffe & Fette & C-Hydrate & Alcohol \\
\hline $500 \mathrm{gr}$ Kartoffeln & 11,0 & & 100 & \\
\hline 120, Butter & 1,1 & 110 & & \\
\hline 80 "Reis & 5,6 & \multicolumn{3}{|c|}{62} \\
\hline 30 \# Fleisch & 6,4 & & & \\
\hline $120 "$ Speck & 3,6 & 90 & & \\
\hline 50, Milch & 2,0 & \multicolumn{3}{|c|}{2,0} \\
\hline 2 Liter Bier & 10,0 & & 120 & 60 \\
\hline $20 \mathrm{gr}$ Caffee & 0,6 & & 80 & \multirow{3}{*}{20} \\
\hline $80 \#$ Zucker & & & & \\
\hline $1 / 4$ Liter Wein & 0,5 & & 2 & \\
\hline & $40,8 \mathrm{gr}$ & $202 \mathrm{~g}$ & $366 \mathrm{gr}$ & $80 \mathrm{gr}$ \\
\hline
\end{tabular}

Urin $1460 \mathrm{cem}$.

$10 \mathrm{ccm}$ entsprechen $2,8 \mathrm{ccm}$ Normalschwefelsäure $=0,0392 \mathrm{gr} \mathrm{N}$.

Gesammtmenge des $\mathrm{N}$ im Urin $5,723 \mathrm{gr}$.

$n$

$"$ " in der Nahrung 6,5 " 28.-29. Juni.

N-haltige Stoffe Fette C-Hydrate Alcohol

$\begin{array}{lrrrr}500 \mathrm{gr} \text { Kartoffeln } & 11,0 & & 100 & \\ 200 \text { "Butter } & 1,8 & 184 & & \\ 80 \text { " Reis } & 5,6 & & 69 & \\ 21 / 2 \text { Ltr. Bier (1 Ltr. Export) } & 14,5 & & 150 & \\ 20 \mathrm{gr} \text { Caffee } & 0,6 & & 24 & \\ 40 \text { "Brod } & 4,0 & & & \\ 60 \text { "Zucker } & & & 60 & \\ & -37,5 \mathrm{gr} & 184 \mathrm{gr} & 396 \mathrm{gr} & 85 \mathrm{gr} .\end{array}$

Urin $1850 \mathrm{ccm}$.

$10 \mathrm{~cm}$ entsprechen $2,0 \mathrm{~cm}$ Normalschwefelsäure $=0,028 \mathrm{gr} \mathrm{N}$.

Gesammtmenge des $\mathrm{N}$ im Urin $5,18 \mathrm{gr}$.

$"$ " in der Nabrung 6,0 ,

29.-30. Juni.

$500 \mathrm{gr}$ Kartoffeln

N-haltige Stoffe Fette

C-Hydrate Alcohol

150 " Butter

11,0

100

80 "Reis

1,4

138

50 "Speck

5,6

62

$1 \mathrm{Ei}$

1,5

37

$20 \mathrm{gr}$ Caffee

6,0

5

2 Ltr. Bier ( $1 / 2$ Ltr. Export) 11,0

120

65

$90 \mathrm{gr}$ Zucker

90

$371 \mathrm{gr} \quad 180 \mathrm{gr} \quad 372 \mathrm{gr} \quad 65 \mathrm{gr}$.

Urin $1840 \mathrm{ccm}$. 
Untersuchungen über den Eiweissbedarf des Menschen.

$10 \mathrm{~cm}$ entsprechen $1,8 \mathrm{~cm}$ Normalschwefelsäure $=0,0252 \mathrm{gr} \mathrm{N}$. Gesammtmenge des $\mathrm{N}$ im Urin $4,636 \mathrm{gr}$.

" " in der Nahrung 5,93 "

I. Versuchseihe.

Aufgenommener N. Abgegebener N.

\begin{tabular}{|c|c|c|}
\hline 1. $\mathrm{Tag}$ & 6,72 & 9,604 \\
\hline 2. " & 7,15 & 7,469 \\
\hline 3. & 7,25 & 6,756 \\
\hline 4. & 6,64 & 5,52 \\
\hline 5. & 6,18 & 5,71 \\
\hline 6. & 7,52 & 5,32 \\
\hline 7. & 6,3 & 5,387 \\
\hline 8. & 4,4 & 4,9 \\
\hline 9. & 5,56 & 3,92 \\
\hline 10. & 5,44 & 4,41 \\
\hline 11. & 6,2 & 6,048 \\
\hline 12. & 5,3 & 4,984 \\
\hline 13. & 6,5 & 5,723 \\
\hline 14. & 6,0 & 5,18 \\
\hline 15. & 5,93 & 4,636 \\
\hline
\end{tabular}

Die durchschnittliche $\mathrm{N}$-Aufnahme vom $4 .-15$. Tage war $6,0 \mathrm{gr} \mathrm{N}$.

Die N-Abgabe war 5,14 gr N.

Durchschnittswerth der Nahrung an den einzelnen Tagen:

$\begin{array}{lr}\text { an N-haltigen Stoffen } 38,89 \mathrm{gr} \\ \text { an Fetten } & 172,6 " \\ \text { an Kohlehydraten } & 358,3 \% \\ \text { an Alcohol } & 74,2,\end{array}$

II. Versuchsreihe.

\begin{tabular}{|c|c|c|c|c|}
\hline $500 \mathrm{gr}$ Kartoffeln & 11,0 & \multicolumn{3}{|c|}{100} \\
\hline 180, Butter & 1,6 & 165 & & \\
\hline 120 \# Reis & 8,4 & \multicolumn{3}{|c|}{94} \\
\hline $40 \#$ Semmel & 4,0 & \multicolumn{3}{|c|}{24} \\
\hline 2 Ltr. Bier (1 Ltr. Export) & 12,0 & \multirow{2}{*}{\multicolumn{2}{|c|}{120}} & 70 \\
\hline $20 \mathrm{gr}$ Caffee & 0,6 & & & \\
\hline 50, Milch & 2,0 & 2,0 & 2,0 & \\
\hline 80 ZZucker & & \multicolumn{3}{|c|}{80} \\
\hline & $39,6 \mathrm{~g}$ & $167 \mathrm{gr}$ & $420 \mathrm{gr}$ & $70 \mathrm{gr}$ \\
\hline
\end{tabular}

Urin konnte in Folge eines Versehens nicht bestimmt werden.

Menge des $\mathrm{N}$ in der Nahrung 6,3 gr.

E. PAlüger, Archiv f. Physiologie. Bd. XII. 


$$
\text { 10.-11. Juli. }
$$

$\begin{array}{ccc}\text { N-haltige Stoffe } & \text { Fette } & \text { C-Hydrate } \\ 11,0 & 100 & \text { Alcohol }\end{array}$

500 gr Kartoffeln

150 "Butter

2 Ltr. Bier (1 Ltr. Export) 12,0

$120 \mathrm{gx}$ Reis

90 "Zucker

20 "Caffee

40 " Semmel
138

$1,4 \quad 138$

70

94

90

Urin $1230 \mathrm{ccm}$.

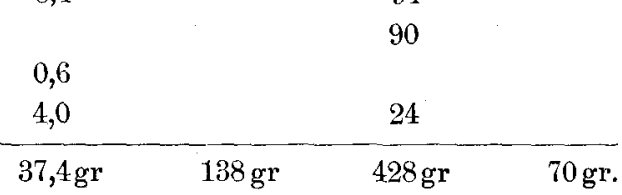

$10 \mathrm{ccm}$ entsprechen $4,6 \mathrm{ccm}$ Normalschwefelsäure $=0,0644 \mathrm{gr} \mathrm{N}$.

Gesammtmenge des $\mathrm{N}$ im Urin 7,921 gr.

" $"$ in der Nahrung 6,0 "

11.-12. Juli.

N-haltige Stoffe Fette C-Hydrate Alcohol

\begin{tabular}{|c|c|c|c|c|}
\hline $500 \mathrm{gr}$ Kartoffeln & 11,0 & \multicolumn{3}{|c|}{100} \\
\hline $200 "$ Butter & 1,8 & 184 & & \\
\hline $120 "$ Reis & 8,4 & \multicolumn{3}{|c|}{94} \\
\hline $40 "$ Brod & 4,0 & \multicolumn{3}{|c|}{24} \\
\hline 2 Ltr. Bier (1 Ltr. Export & 12,0 & \multirow{2}{*}{\multicolumn{2}{|c|}{120}} & \multirow[t]{3}{*}{70} \\
\hline $20 \mathrm{gr}$ Caffee & 0,6 & & & \\
\hline $70 .$, Zucker & & \multicolumn{2}{|r|}{70} & \\
\hline $1 / 4$ Liter Wein & 0,5 & & 2 & 20 \\
\hline & $38,3 \mathrm{gl}_{1}$ & $184 \mathrm{gr}$ & $410 \mathrm{gr}$ & $90 \mathrm{gr}$ \\
\hline
\end{tabular}

Urin $1620 \mathrm{ccm}$.

$10 \mathrm{ccm}$ entsprechen $3,0 \mathrm{ccm}$ Normalschwefelsäure $==0,042 \mathrm{gr}$.

Gesammtmenge des $\mathrm{N}$ im Urin $\quad 6,804 \mathrm{gr}$.

" " $\quad$ in der Nahrung 6,1 "

12.-13. Juli,

$\begin{array}{lcccc} & \text { N-haltige Stoffe } & \text { Fette } & \text { C-Hydrate } & \text { Alcohol } \\ 500 \mathrm{gr} \text { Kartoffeln } & \mathbf{1 1 , 0} & & 100 & \\ 210 \text { "Butter } & 1,9 & 193 & & \\ 120 \text { " Hirse } & 13,9 & & 84 & \\ 40 \text { "Semmel } & 4,0 & & 24 & 70 \\ 2 \text { Ltr. Bier (1 Ltr. Export) } & 12,0 & & 120 & \\ 20 \mathrm{gr} \text { Caffee } & 0,6 & & 80 & 80 \\ 80 \text { "Zucker } & & & 408 \mathrm{gr} & 70 \mathrm{gr} .\end{array}$

Urin $1530 \mathrm{ccm}$. 
$10 \mathrm{ccm}$ entsprechen $2,6 \mathrm{ccm}$ Normalschwefelsäure $=0,0364 \mathrm{gr} N$.

Gesammtmenge des $\mathrm{N}$ im Urin $\quad 5,569 \mathrm{gr}$.

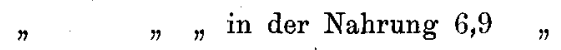

$$
\text { 13.-14. Juli. }
$$

$\begin{array}{lcccc} & \text { N-haltige Stoffe } & \text { Fette } & \text { C-Hydrate } & \text { Alcohol } \\ 500 \mathrm{gr} \text { Kartoffeln } & 11,0 & & 100 & \\ 220 \text { " Butter } & 2,0 & 202 & & \\ 50 \text { " Reis } & 3,5 & & 39 & \\ 80 \text { " Semmel } & 8,0 & & 48 & \\ 2 \text { Ltr. Bier (1 Ltr. Export) } & 12,0 & & 120 & 70 \\ 20 \text { gr Caffee } & 0,6 & & 80 & \\ 80 \text { "Zucker } & & & & \\ & 37,1 \mathrm{gr} & 202 \mathrm{gr} & 387 \mathrm{gr} & 70 \mathrm{gr} .\end{array}$

Urin $1050 \mathrm{ccm}$.

$10 \mathrm{ccm}$ entsprechen $3,2 \mathrm{ccm}$ Normalschwefelsäure $=0,0448 \mathrm{gr}$ N.

Gesammtmenge des $\mathrm{N}$ im Ürin $4,704 \mathrm{gr}$.

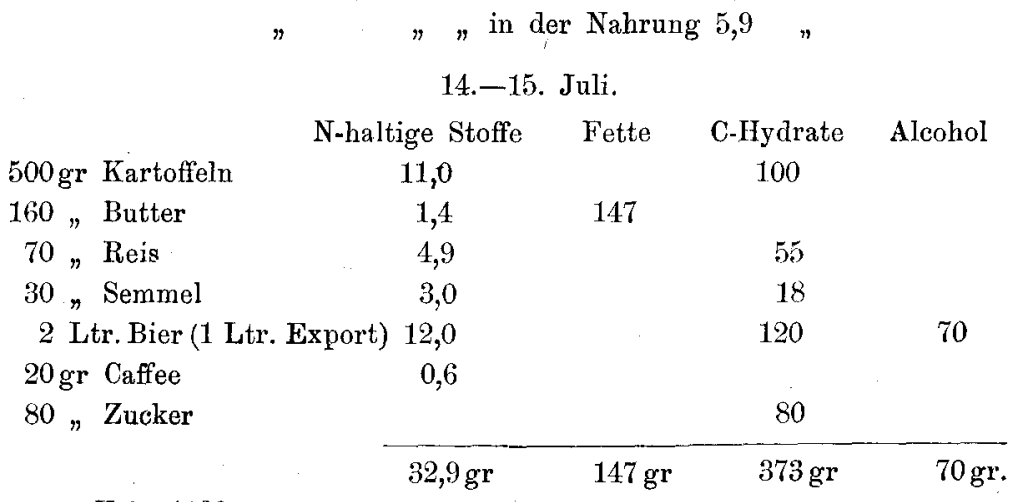

Urin $1120 \mathrm{ccm}$.

$10 \mathrm{~cm}$ entsprechen $2,4 \mathrm{~cm}$ Normalschwefelsäure $=0,0336 \mathrm{gr}$ N.

Gesammtmenge des $\mathrm{N}$ im Urin $\quad 3,763 \mathrm{gr}$.

" $"$ in der Nahrung 5,3 "

15. - 16. Juli.

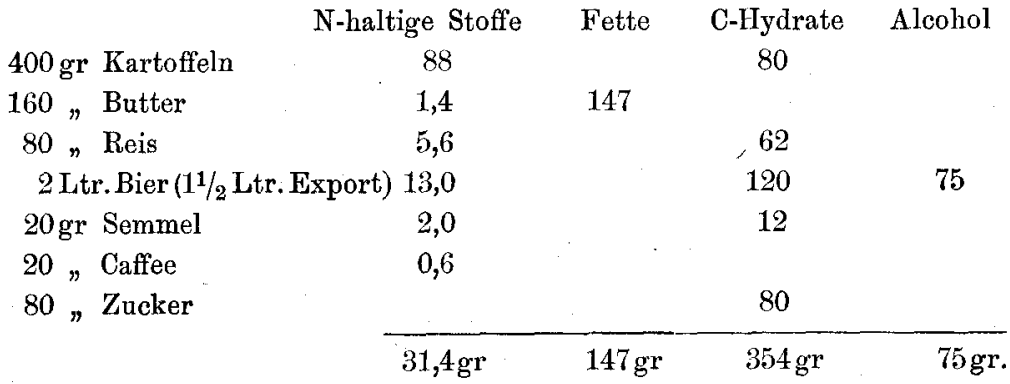


Urin $1200 \mathrm{ccm}$.

$10 \mathrm{ccm}$ entsprechen $2,8 \mathrm{ccm}$ Normalschwefelsäure $=0,0392 \mathrm{gr} \mathrm{N}$.

Gesammtmenge des $\mathrm{N}$ im Urin

$4,704 \mathrm{gr}$.

" , in der Nahrung 5,0 " 16.-17. Juli.

\begin{tabular}{|c|c|c|c|c|}
\hline & N-haltige Stofte & Fette. & C-Hydrate & Alcohol \\
\hline $400 \mathrm{gr}$ Kartoffeln & 8,8 & & & \\
\hline $200 "$ Butter & 1,8 & 184 & & \\
\hline 1 Liter Exportbier & 7,0 & & 60 & 40 \\
\hline $1 / 2 \quad n \quad$ Wein & 1,0 & & 5 & 40 \\
\hline $60 \mathrm{gr}$ Reis & 4,2 & & 148 & \\
\hline 180, Brod & 18,0 & & 108 & \\
\hline 30 \# Speck & 0,9 & 22 & & \\
\hline 20 „Caffee & 0,6 & & & \\
\hline 120 ,Zucker & & & 120 & \\
\hline & $42,3 \mathrm{gr}$ & $206 \mathrm{~g}$ & $420 \mathrm{gr}$ & $80 \mathrm{gr}$ \\
\hline
\end{tabular}

Urin $1190 \mathrm{ccm}$.

$10 \mathrm{ccm}$ entsprechen $3,3 \mathrm{ccm}$ Normalschwefelsäure $=0,0462 \mathrm{gr} \mathrm{N}$.

Gesammtmenge des $\mathrm{N}$ im Urin $\quad 5,497 \mathrm{gr}$.

" " in der Nahrung 6,8 " 17.-18. Juli.

$600 \mathrm{gr}$ Kartoffeln

200 , Butter

110 "Semmel

$1 \mathrm{Ei}$

2 Liter Bier

$20 \mathrm{gr}$ Caffee

90 \#Zucker
Fette

N-haltige Stoff

13,2

1,8

11,0

6,0

10,0

0,6
C-Hydrate Alcohol

120

184

66

5

120

60

Urin $1080 \mathrm{ccm}$.

$-\frac{90}{42,6 \mathrm{gr}}-189 \mathrm{gr} \quad-\frac{396 \mathrm{gr}}{60 \mathrm{gr} .}$

$10 \mathrm{ccm}$ entsprechen $3,7 \mathrm{~cm}$ Normalschwefelsäure $=0,0518 \mathrm{gr} \mathrm{N}$.

Gesammtmenge des N im Urin $5,5944 \mathrm{gr}$.

$" \quad "$ in der Nahrung 6,8 n 18.-19. Juli.

\begin{tabular}{|c|c|c|c|c|}
\hline $400 \mathrm{gr}$ Kartoffeln & 8,8 & \multicolumn{3}{|c|}{80} \\
\hline 180 "Butter & 1,6 & 166 & 72 & \\
\hline 120, Brod & 12,0 & & 39 & \\
\hline 50 , Reis & 3,5 & & & \\
\hline 2 Ltr. Bier (1 Ltr. Export) & 12,0 & & 120 & 70 \\
\hline 80 gr Zucker & & & 80 & \\
\hline 20 , Caffee & 0,6 & & & \\
\hline & $38,5 \mathrm{~g}$ & $166 \mathrm{~g}$ & $391 \mathrm{gr}$ & $70 \mathrm{gr}$ \\
\hline
\end{tabular}


Urin $1180 \mathrm{ccm}$.

$10 \mathrm{ccm}$ entsprechen $3,2 \mathrm{~cm}$ Normalschwefelsäure $=0,0448 \mathrm{gr} \mathrm{N}$.

Gesammtmenge des $\mathrm{N}$ im Urin $\quad 5,286 \mathrm{gr}$.

$" \quad, \quad$ in der Nahrung 6,2 ",

II. Vers u chs reibe.

Aufgenommener N. Abgegebener N.

$\begin{array}{ll}6,3 \mathrm{gr} & \\ 6,0 & 7,921 \mathrm{gr} \\ 6,1 & 6,804 \\ 6,9 & 5,569 \\ 5,9 & 4,704 \\ 5,3 & 3,763 \\ 5,0 & 4,704 \\ 6,8 & 5,497 \\ 6,8 & 5,594 \\ 6,2 & 5,286\end{array}$

Die durchschnittliche N-Aufnahme vom 4.-10. Tage war $=6,11 \mathrm{gr} \mathrm{N}$.

Die N-Abgabe war $5,01 \mathrm{gr} \mathrm{N}$.

Durchschnittswerth der Nahrung an den einzelnen Tagen:

an N-haltigen Stoffen $38,4 \mathrm{gr}$.

$\begin{array}{lr}\text { an Fetten } & 173,9, \\ \text { an Kohlehydraten } & 398,7, \\ \text { an Alcohol } & 72,5,\end{array}$

Fasst man nach diesen Zahlen den Gang der Eiweisszersetzung zuerst ins Auge, so findet man:

Die im Urin ausgeschiedene N-Menge übersteigt nur an den beiden ersten Tagen, im zweiten Versuche an den drei ersten Tagen, die aufgenommene. Der Organismus setzt sich dann mit der eingeführten Eiweissmenge ins Gleichgewicht, das er am dritten beziehungsweise vierten Tag erreicht zu haben seheint. Dasselbe wiederholte sich noch einmal, als in der ersten Versuchsreihe die eingeführte $\mathrm{N}$-Menge von 6,3 auf $4,4 \mathrm{gr}$ herabgesetzt wurde. Aehnlich sind die Zahlen der zweiten Versuchsreihe. Man kann dies, wenn man den von Voit ${ }^{1}$ ) angegebenen Gang der Eiweisszersetzungen verfolgt, leicht erklären.

Die der Ernährung mit 100-120gr Eiweiss entsprechende Menge von circulirendem Eiweiss ging, sowie diese Kost nicht

1) Vo it, Handbuch des Stoffwechsels S. 303 u. folg. 
mehr geboten wurde, allmählich in Verlust. Es blieb am Körper nur so viel Eiweiss - natürlich eirculirendes, nicht Organeiweiss wie viel der geringeren Eiweissmenge der Nahrung entsprach. Dabei ist zu beachten, dass der gesammte $\mathrm{N}$ unserer Nahrung nicht in Eiweiss enthalten war! Wenn man vom $\mathrm{N}$ des Caffein absieht, so ist doch wichtig, dass in den Kartoffeln durchschnittlich nur $50-70 \%$ der N-haltigen Stoffe Eiweiss sind ${ }^{1}$ ), den übrigen Theil machen Amidokörper, Peptone u. s. w. aus.

Dasselbe Verhalten, $d$. h. die Abgabe von circulirendem Eiweiss bei nicht geniigender Neueinfuhr ist dann wieder zu beobachten, als in der ersten Versuchsreihe die Menge an N-haltigen Substanzen von 6,3 anf $4,4 \mathrm{gr}$ vermindert wurde.

Ob mit letzterer Nahrungsmenge, die an Eiweissstoffen nicht mehr als 20-25 gr enthielt, das Minimum an Eiweiss bestimmt ist, mit dem der erwachsene Mensch auskommt, ist nicht zu entscheiden. Jedenfalls geht die N-Ausscheidung in unseren Versuchen ebenso wie bei reichlicher Ernährung der N-Aufnahme deutlich parallel. Dadurch allein wird schon die Annahme kaum denkbar, dass während der Versuchszeit eine Degeneration von Körpereiweiss stattgefunden hätte, welche doch auf die Grösse der NAusscheidung einen sicher leicht nachweisbaren Einfluss ausgeübt haben würde. Auch der Umstand, dass die körperliche Leistungsfähigkeit, wie erwähnt, nicht herabgesetzt, sondern eher vermehrt war, spricht sehon dagegen. Der Einwand aber, dass zersetzte organische Bestandtheile, besonders Harnstoff im Körper zurückgehalten wurden, ist schon deshalb nicht annehmbar, weil die $\mathrm{N}$ Ausscheidung am ersten Tage mit eiweissreicher Kost trotz reichlichen Trinkens durchaus nicht einmal der eingeführten N-Menge entsprechend vermehrt war. Auch scheint nach allen Untersuchungen Voits die Möglichkeit eines solchen Zurückbleibens Nhaltiger Produkte im Körper ausgeschlossen.

Die Fette wurden täglich in einer Menge von ca. $170 \mathrm{gr}$ genossen. Es fanden dabei allerdings ziemlich bedeutende Schwankungen an den einzelnen Tagen statt. Die Menge scheint ziemlich beträchtlich und ibertrifft das gewohnte Tagesquantum gewiss bedeutend, doch findet man in der Nahrung einzelner Arbeiter oft noch böhere Werthe ${ }^{2}$ ).

1) Schultze u. Engster, citirt nach M a ly, Jahresber. XII, 460.

2) V o it, Handbuch des Stoffwechsels S. 524. 
Von den Kohlehydraten wurden täglich meist $360-400 \mathrm{gr}$ verzehrt und wenn man die im Bier enthaltenen nicht mit in Rechnung zieht, meist ca. $250 \mathrm{gr}$. Es sind dies geringere Quantitäten als die $500 \mathrm{gr}$, die von Voit ${ }^{1}$ ) für den Arbeiter als zulässig erachtet werden und bedeutend weniger als von einigen Beobachtern ${ }^{2}$ ) bei sich wesentlich von Vegetabilien nährenden Personen gefunden wurden.

Das Gewicht der täglichen Nahrung schwankte zwischen 1000 und $1400 \mathrm{gr}$, wenn man dabei von der rein flüssigen Kost: Milch, Bier, Wein absieht. Das Volumen wurde nicht bestimmt, doch schien es ungefähr dem der gewohnten Nahrung zu entsprechen oder übertraf es nur wenig.

Die durch den Genuss aller Nahrungsmittel im Organismus erzeugten Calorien betrugen, wenn man die von $R u b n$ er ermittelten Werthe

$$
\begin{aligned}
& 1 \mathrm{gr} \text { Eiweiss }=4100 \text { Calor. (ohne Harnstoff) } \\
& 1, \text { Fett }=0300 \quad, \\
& 1, \text { Kohlehydr. }=4100 \quad,
\end{aligned}
$$

einträgt, bei der ersten Versuchsreihe ca. 3750 , bei der zweiten 3916 Calorien. Der Verbrennungswerth des Alcohol ist $1 \mathrm{gr}$ gleich 7000 Calorien gerechnet. Nimmt man als durch mangelnde Resorption verloren gegangen $10 \%$ an, so bleibt ein Durehschnittswerth von ca. 3500 Calorien. Es entspricht diese Zahl ungefähr den von $R u b n e r$ für Arbeiter, die mittelschwere Arbeit $z u$ leisten haben, gefundenen Ziffern. Der dabei von den Kohlehydraten und Fetten gelieferte Antheil betrug je ca. $40 \%$, während in der gewöhnlichen Nahrung der Arbeiter die Kohlehydrate meist $60 \%$, die Fette nur ca. 20\% der Wärme liefern. Der vom Alcohol gelieferte Theil betrug hier fast 15, der des Eiwoiss in der ersten Versuchsreihe $4,2 \%$, in der zweiten $4,0 \%$, während er bei gewöhnlicher Ernährungsweise wohl 15-20\% ausmacht. Dabei sind aber die gesammten $\mathrm{N}$-haltigen Stoffe gerechnet, die allein von Eiweiss gelieferten Wärmemengen werden daher noch geringer sein!

Wie lässt sich nun dieses Resultat, dass erst 15 Tage, dann

1) Voit, ebenda S. 524-525.

2) Voit, Handbuch des Stoffwechsels S. 524.

3) R u b n er, Zeitschr. für Biolog. XIX u. XXI, vergl besond. Maly, Jahresbericht XV 394 u. folg. 
nach 8tägiger Pause 10 Tage N-Gleichgewicht mit so geringen Eiweissmengen in der Nahrung vorhanden war, mit der herrschenden Ansicht die auf so viel genau beobachteten Versuchen basirt, in Einklang bringen?

Dabei ist nun die erste Frage. Ist die bei längerem Hunger annähernd constant ausgeschiedene N-Menge, das sogenannte typische Hungerminimum ein Maass für die geringste Menge Eiweiss, welche der Organismus nöthig hat, um sich auf seinem Bestande zu erhalten? Von mehreren Forschern ist diese Frage in der That bejaht worden. Doch wären Einwände hiergegen zu erheben. Beim Hunger zerfällt in Folge der mangelnden Zufuhr an Ernährungsmaterial überhaupt allmählich ein grosser Theil des Körpereiweisses. Dass das gerade so viel ist, als die Organe bei der gewöhnlichen Ernährung ergänzen, ist möglich, jedoch nicht bewiesen. Jedenfalls weist die Thatsache, dass einzelne Organe, z. B. das Gehirn und Herz beim Hunger sebr wenig verlieren auf Thatsachen hin, welche dies compliciren. Nach $V_{0}$ it $^{1}$ ) lässt sich dieser Vorgang in folgender Weise deuten:

Das Eiweiss der Organe schmilzt im Hunger als solches ab, gelangt in Lösung in den Säftestrom und dient dann zur Ernährung anderer Organe, z. B. des Gehirns, Rückenmarks, der Eierstöcke und Hoden beim Lachs u. s. w. oder es wird zersetzt.

Auch wird die Thatsache, dass bei N-loser Kost die N-Ausscheidung bedeutend geringer wird, als beim Hunger nur verständlich, wenn man erwägt, dass es allein der Mangel an Nabrung ist, warum der $\mathrm{N}$-haltige Atomencomplex des Organismus zerfällt. Mit mehr Recht könnte man daher noch annehmen, dass der normale Eiweissbedarf durch die bei reichlicher N-loser Kost ausgeschiedene N-Menge bestimmt ist. Jedenfalls giebt die im Hunger ausgeschiedene N-Menge keinen Maassstab für die Eiweissmenge $a b$, welche der Organismus nöthig hat, um sich auf seinem Bestande zu erhalten.

Eine weitere wichtige Frage ist, welchen $Z_{\text {weck }}$ und welche Vortheile bietet eine reichliche Eiweissernährung. Zur Entscheidung dieser Frage ist vor allem wichtig, das Verhalten des Organismus dabei kennen zu lernen. Nach einer eiweissreichen Mahl-

1) V o it, Handbuch des Stoffwechsels S. 303. 
zeit ist, wie Voit $\left.{ }^{1}\right)$ hervorhebt, ,zu einer Stunde in der die Verdauung noch im vollen Gange ist, schon mindestens die Hälfte des im Magen aufgenommenen Eiweisses zerstört und der Stickstoff desselben aus dem Körper ausgestossen." Voit fand bei dem Menschen nach einer reichlich aus Fleiseh und Eiern bestehenden Mahlzeit die N-Ausscheidung im Harn schon nach einer Stunde zunehmen, in 7 Stunden war das Maximum erreicht, um in den nächsten 10 Stunden allmäblich abzunehmen. Aehnliche Versuche stellten $\mathrm{Panum}^{2}$ ) und $\mathrm{Feder}{ }^{3}$ ) an Hunden an. Panum fand das Maximum der $\mathrm{N}$-Ausscheidung schon nach 3-6 Stunden erreicht, in 7 Stunden war die Hälfte der N-Menge secernirt, welche nach Aufnahme der betreffenden Fleischportion in 24 Stunden ausgeschieden wird. Die Resultate unserer Versuche stimmten hiermit ziemlich genau. Wir fanden nach einer Mahlzeit die aus $300 \mathrm{gr}$ Fleisch, $40 \mathrm{gr}$ Fett und $150 \mathrm{gr}$ Semmel bestand, also ca. 12,5 gr N enthielt, das Maximum der $\mathrm{N}$-Ausscheidung in der 3.-5. Stunde. Wurde die Mahlzeit um $100 \mathrm{gr}$ Fleisch (= ca. 3,4 gr N) vermehrt, so war in den ersten $4-5$ Stunden die N-Ausfuhr die gleiche, aber in der 6. Stunde erfolgte kein Absinken, sondern in den beiden nächsten Stunden wurden noch annähernd dieselben Mengen ausgeschieden wie in der 3.-5. Stunde. Aehnliche Ergebnisse batten die von Feder an Hunden gemachten Beobachtungen. Derselbe fand, wenn die Fleischmahlzeit, statt aus $500 \mathrm{gr}$ ans $1000 \mathrm{gr}$ Fleisch bestand, bald in den ersten Stunden ein bedeutendes Ansteigen, wenn auch nicht in dem Verhältniss wie es der um das doppelte gesteigerten Nahrung entsprochen hätte. Ferner konnte er deutlicb eine verlängerte Periode der Ausscheidung grösserer N-Mengen constatiren. Es verbält sich demnach die Elimination des iberschüssigen Eiweisses wiẹ die des Leims. Nur ist der Untersehied, dass bei reichlicher Eiweissnahrung immer eine der Ernährung entsprechende Menge von circulirendem Eiweiss im Körper angehäuft wird, während der Leim sofort zerstört und entfernt wird.

Dieser Vorgang macht durchaus den Eindruck, als wenn der Organismus bei der Eiweisszersetzung nur im Auge habe: sich

1) Voit, Handbuch des Stoffwechsels S. 107 u. 108.

2) $\mathrm{P}$ a n u m, citirt nach M a $1 \mathrm{y}$, Jahresbericht IV, S. 365.

3) F ed e r, Zeitschr. für Biologie XVII, S. 531. 
der N-haltigen Atomcomplexe möglichst rasch zu entledigen. Vo it betonte allerdings in Wirdigung dieser Thatsachen, welche eine Hauptstütze für die sogenannte Luxustheorie bildeten, dass es eine Luxuszersetzung von Eiweiss doch nicht gebe. Reichlich N-haltige Nahrung bedinge einen hoben Eiweissstand des Körpers, der dann durch reichliche albuminathaltige Kost unterhalten werden müsse.

„Nur, so führt er aus ${ }^{1}$ ), wenn die dureh den Umsatz herbeigeführte Leistungsfähigkeit ein Luxus ist, dann ist auch der grössere Umsatz ein Luxus gewesen."

Dieser Satz, der bis jetzt fïr einen der wichtigsten Grundsätze der heutigen Ernährungslebre gilt, legt die Frage nahe, in welcher Beziehung steht die reichliche Eiweissnahrung zu der vermehrten Leistungsfähigkeit des Organismus. Inwieweit ist durch die bisherigen Versuche ein solches Verhältniss sichergestellt?

Sehr einfach scheint dabei der Hinweis, dass Jeder, der kräftige Arbeit leistet, schliesslich eine starke Muskulatur, d. i. eine Vermehrung des eiweissreichsten Organsystems bekommt. In dem Sinne ist es auch einleuchtend, dass derselbe auch mehr Eiweiss zur Unterhaltung seiner grossen Körpermasse nothwendig hat als der, welcher wenig körperlich arbeitet, also eine bedentend geringere Muskelmasse besitzt. Dennoch lassen sich hiergegen Einwände erheben, besonders wenn dieser Satz, wie es häufig geschieht, geltend gemacht wird, um z. B. die Nothwendigkeit von 100-120 gr Eiweiss in der täglichen Nahrung einzelner Arbeiterklassen zu beweisen.

Der erste Einwurf ist, dass eigentlich noch nicht sicher bestimmt ist, wie viel Eiweiss für eine gewisse Körpermasse nothwendig ist. Es sind also beide Factoren in diesem Verhältniss unbekannt.

Um die eiweissreichere Nahrung muskelkräftiger Individuen in dieser Weise zu erklären, mlisste man annehmen, dass immer ein Theil der Muskulatur abgenutzt und ersetzt wird. Je mehr Muskulatur am Körper ist, um so höher müsste dann daher dieser Factor ausfallen.

Vorzüglich aber Voit betonte hiergegen, dass eine so fortwährende Auswechselung des neu eingeführten Eiweisses mit dem Zelleneiweiss unwahrseheinlich sei.

1) V o it, Zeitschr. für Biologie III, S. 39. 
Nur die Blutkörperchen und einzelne Epithelien gehen theilweise fortwährend in Verlust und werden in demselben Maasse nengebildet. Bei den meisten anderen Geweben, wo das Wachsthum sehr langsam vor sich geht, ist zu diesem Zweck sicher sehr wenig Eiweiss unter gewöhnlichen Verhältnissen nöthig. Ueber die Grösse dieses Bedarfs haben wir allerdings keine genaue Erfahrung, doch durfte er, wie Voit) mit Recht bei Besprechung der Ernährung mit Leim ausführt, sehr wahrscheinlich nicht bedeutend anzuschlagen sein. Dieser Factor wird allerdings bei verschieden grossen Individuen die verschiedene Arbeit leisten und demgemäss eine verschiedene Muskulatur haben, auch dementsprechend immer anders ausfallen. Doch wird der grösste Theil des Nahrungseiweiss durchaus nicht zu diesem $Z$ wecke verwandt. Der Gang der Eiweisszersetzung ist vielmehr wesentlich der ${ }^{2}$ ).

Das aufgenommene Eiweiss geräth in den Säftestrom und vergrössert damit die Eiweisszersetzung. Zugleich wird dadurch die Menge des circulirenden Eiweisses im Körper vermehrt. Durch einen solchen reichlichen Strom von circulirendem Eiweiss wird aber nach den meisten Ansichten erstens die Leistungsfähigkeit des Organismus erhöht und zweitens verhütet, dass das Organeiweiss verloren geht. Letztere Anschauung geht dahin aus. Sobald die Menge des circulirenden Eiweisses unter eine gewisse Norm sinkt (dies tritt bei einem erwachsenen Mann bei einer Nahrung ein, die nach $V_{0}$ it $^{3}$ ) weniger als 108 beziehungsweise $118 \mathrm{gr}$ Eiweiss enthält), schmilzt das Organeiweiss ab, d. h. gelangt in die Säfte, wird zersetzt und ausgeschieden.

Wie lassen sich nun diese beiden Ansichten durch das Ergebniss sicherer Versuche oder durch theoretische Erwägungen vertheidigen oder beweisen?

Zuvörderst entspricht es durchaus unsern Anschauungen und liegen hierfür reichlich Beweise vor, dass Leute, welche sich hauptsächlich von den eiweissreichen Nahrungsmitteln, besonders Fleisch nähren, auch ausserordentlich leistungsfähig sind. Dass dies jedoch nicht immer so ist, wird wohl Jedermann schon beobachtet

1) Voit, Handbuch des Stoffwechsels S. 400 u. 276.

2) Voit, ebenda S. 107 u. 108 u. 303 u. folg.

3) Voit, Handbuch des Stoffwechsels S. 525 u. Zeitschr. f. Biologie $\mathrm{XV}$, S. 459 . 
haben. Man sieht so häufig Fabrik- oder ländliche Arbeiter die bedeutendsten Anstrengungen ausführen und dabei nur oder wenigstens vorziglich Kartoffeln und Schnaps geniessen. Wir sind allerdings in diesen Fällen geneigt, alle Störungen bei den letztgenannten Klassen als Folgen der ungenügenden Eiweissnahrung zu betrachten. Doch ist dabei zu bedenken, dass diese Leute in Folge ihrer Armuth die eiweissreichen Nahrungsmittel, welche, wie noch später erwähnt wird, bei weitem die theuersten sind, sich nicht beschaffen können. Thre Nahrung ist daher auch meist schlecht zubereitet und leiden sie an so vielen hygienischen Missständen, dass wir darin allein eine Erklärung für einen etwaigen sehlechten Körperstand finden können.

Wie wäre ferner ein Zusammenhang zwischen der Eiweisszersetzung der Zelle und ihrer Arbeitsleistung denkbar? Sicher nachgewiesen ist, dass die N-Ausscheidung des Organismus durch die Muskelthätigkeit nicht wesentlich beeinflusst wird. Wir könnten also nicht einmal annehmen, dass reichliche Mengen circulirenden Eiweisses dem Körper als eine Art Brennmaterial dienen. Nur die N-freien Stoffe werden bei der Muskelthätigkeit in erhöhtem Maasse in Zersetzung gezogen. Wenn nun diese Stoffe auch aus den Albuminaten entstehen können, so ist es doch wohl näherliegend dem Organismus die ersteren selbst zuzuführen.

Es wäre nun der zweite Punkt zu untersuchen. Ist das Organeiweiss in Gefabr, durch eine etwas zu eiweissarme Kost abgeschmolzen zu werden, $d$. h. in den Verlust zu gehen?

Hamilton E. Bowie, der in Voit's Laboratorium arbeitete, behauptet ${ }^{1}$ ):

„Der Arbeiter würde durch Entziehen von Eiweiss an einem Rubetage oder Sonntag an Muskelmasse verlieren und dann am Montag nicht mehr die gewohnte Arbeit leisten."

Dernnach müsste eigentlich ein kräftiger Arbeiter, der aus irgend welchem Grunde ein bis zwei Tage wenig Nahrung oder nur wenig Eiweiss essen könnte, am dritten Tage nicht eher im Stande sein zu arbeiten, als bis er seinen vollständigen Eiweissvorrath wieder ergänzt hat. Abgesehen von den Versuchen des Verfassers sprechen gegen ein solches rasches Einschmelzen des 
Organeiweiss, das mit raschem Verlust der Körperkräfte gleichbedeutend wäre, auch Untersuchungen anderer Autoren. So fand Parkes ${ }^{1}$ ), dass ein kräftiger Mann, dessen stickstoffhaltige Nahrung 5 Tage hindurch auf die Hälfte reducirt war, der dann noch 5 Tage günzlich stickstofffreie Kost bekam, doch noch am 4. Tage nach dieser Entziehung im Stande war, sehr schwere Arbeit zu leisten und sich ganz wohl dabei befand. Selbstverständlich musste besonders bei letzterer Ernährung der Körperzustand allmählich ein schlechter werden, da, so lange der Organismus lebt, er immer etwas Eiweiss nöthig hat. Noch bestimmter ist ein Versuch Rubner's ${ }^{2}$ ). Derselbe gab einem kräftigen Soldaten 3 Tage lang je $3 \mathrm{~kg}$ Kartoffeln, $100 \mathrm{gr}$ Butter, $20 \mathrm{gr}$ Oel. Rubner bezweckte hierbei, die Resorption grösserer Massen vegetabilischer Nahrung zu untersuchen. Folgende Zahlen von ihm sind bemerkenswerth.

Die N-Ausscheidung der Versuche bei gewöhnlicher Ernählung ist nicht angegeben, sie hat sich wahrscheinlich innerhalb der bekannten Grenzwerthe dieser Klassen, ca. 15-18 gr N, bewegt. Er schied in der Versuchsreihe bei einem durchschnittlichen $\mathrm{N}$-Gehalt der Nahrung von $11,45 \mathrm{gr}$ im Urin aus:

$\begin{array}{rrrr}\text { den } & \text { I. Tag } & 12,8 \mathrm{gr} & \mathrm{N} \\ & \text { II. } & 7,6 . & , \\ " & \text { III. } & 6,0 & \end{array}$

ausserdem in den Fäces in den 3 Tagen zusammen 11,06 gr. Die Ausscheidungen betrugen also $37,5 \mathrm{gr} \mathrm{N}$ gegenüber $34,4 \mathrm{gr}$, die aufgenommen wurden. Es ist also allerdings, wie $\mathrm{Rubner}$ hervorhebt, Eiweiss vom Körper in Verlust gegangen, doch tritt unverkennbar das Bestreben des Organismus hervor, sich ins $\mathrm{N}$ Gleichgewicht zu setzen, das am 3. Tage schon erreicht scheint. Nur die circulirende Eiweissmenge ist vermindert worden. Wenn dabei das Befinden der Versuchsperson kein gutes war, so dürfte dies mancher eher auf die ungehenere Masse der Ingesta, ca. $3 \mathrm{~kg}$, als auf deren geringen $\mathrm{N}$-Gehalt beziehen.

Reichliche Eiweissnahrung allein erhält eine Muskulatur sicher nicht. Vielmehr lehren schon Erfahrungen aus dem täglichen Leben, dass andauernde Ruhe sowohl an einzelnen Gliedern wie an der gesammten Körpermuskulatur unfehlbar auch bei der reich-

1) Par kes, cit. nach $\mathrm{M}$ a $1 \mathrm{y}$, Jahresbericht $\mathrm{I}, \mathrm{S} .290$.

2) Rubner, Zeitschr. für Biologie XV. 
lichsten Ernährung mit Eiweiss zur Inactivitätsatrophie d. i. zum Schwund der Muskulatur führt. Einige Tage eiweissarmer Kost, auch wenn die Menge an Eiweiss sehr gering ist, sind sicher ohne Einfluss anf die Leistungsfähigkeit des Organismus. Ein Zusammenhang zwischen letzterer und der reichlichen Eiweisszersetzung ist noch nicht sicher nachgewiesen.

Bei diesen Verhältnissen könnte man den Nutzen der Eiweissnahrung vielleicht darin sehen, dass der Körper dadurch reicher an Eiweiss wuirde. Dabei konnte man annehmen, dass erst die Menge des circulirenden Eiweisses erhöht würde und dann dieses in Organeiweiss iubergehe ${ }^{1}$ ). Zur Klarlegung dieses Vorgangs dienen hauptsächlich die Versuche Voits. Bei der Ernährung mit möglichst fettfreiem Fleisch gelang es Voit, bei seinem ea. $30 \mathrm{~kg}$ schweren Hunde erst mit ca. $1500 \mathrm{gr}$ Fleisch ein Gleichgewicht zwischen Aufnahme und Abgabe von $\mathrm{N}$, d. h. ein Verhüten von Eiweissverlust am Körper zu erzielen. Gab er mehr Eiweiss, so trat voribergehend eine Minderausscheidung von Harnstoff ein, in den meisten Fällen trat aber bald wieder Gleichgewicht ein, d. h. es wurden alle eingefulhrten Albuminate zersetzt und ausgeschieden. Voit erklärt dies, wie schon mehrfach erwähnt, damit, dass nur der Bestand des Körpers an circulirendem Eiweiss vermehrt wurde. Einen Uebergang von diesem in das wirkliche Organeiweiss, $d . h$. eine schliessliche Zunahme der Organe, konnte er nur dann durchsetzen, wenn er reichlich Fette und Kohlehydrate beifügte. Um diesen Zweek zu erreichen hatte er, wie er hervorhob, nur relativ geringe Mengen Eiweiss nothwendig, wenn er nur grosse Mengen Fett gab.

Dabei wurde jedoch nicht nur weniger $\mathrm{N}$ ausgeschieden, als aufgenommen wurde, sondern auch verhältnissmässig viel Koblenstoff wurde zurickbehalten ${ }^{2}$ ). Dies liess sich nur in der Weise deuten, dass man annahm. der Organismus setzte Fett an. Der Organismus wurde also nur dann erheblich eiweissreicher, wenn er auch zugleich fettreicher wurde. Einen solchen Körperzustand zu erhalten ist allerdings zu gewissen Perioden, z. B. bei der Ernährung von Recunvalescenten erstrebenswerth, bis zu einem gewissen Grade auch physiologisch zu wünschen, doch wird wobl

1) Voit, Handbuch des Stoffwechsels S. 313.

2) Vo it, Handbuch des Stoffwechsels S. 130 u. folg. 
feststehen, dass man ein solches Ziel nicht immer bei der Ernäh. rung vor Augen haben darf.

Auffallend neben meinen Versuchen, nach denen auf einige Zeit ein $\mathbf{N}$-Gleichgewicht mit verhältnissmässig sebr geringen Mengen Eiweiss möglich ist und es wahrscheinlich erscheint, dass sich dies noch weiter ausdehnen lasse, bleibt die Beobachtung, dass so häufig in der Nahrung der verschiedensten Bevölkerungsklassen grössere Mengen Eiweiss gefunden wurden. Es wäre in der That merkwürdig, dass immer ein solcher Luxus begangen wäre. Denn die Albuminate werden nicht vollständig im Körper ausgenützt, sondern der Harnstoff, der von ibnen abgespalten wird, geht dem Organismus verloren. Sie stehen daher, was den Wärmewerth angeht, mit den Kohlehydraten auf gleicher Stufe und tief unter den Fetten. Dabei sind sie bei Weitem die theuersten von allen Nahrungsstoffen, besonders das thierische Eiweiss. So liefern z. B. $500 \mathrm{gr}$ knochenfreien mageren Rindfleisches, die ca. 70 Pfennig kosten, mit ca. $106 \mathrm{gr}$ Eiweiss nur ca. 435 Calorien. Für dieselbe Summe erhält man aber ca. $300 \mathrm{gr}$ Butter, die mit $276 \mathrm{gr}$ Fett ca. 2566 Calorien liefern, deren physiologischer Nutzeffect, was die Wärmeentwicklung angeht, also ca. 6 mal so gross ist. Bei den noch billigeren thierischen Fetten, die, wie besonders das Schweinefett, von den ärmeren Klassen mit Vorliebe genossen werden, tritt dieses Missverhältniss noch mehr hervor.

Es könnte demnach nahe liegen anzunehmen, dass auf die Daner der Organismus mehr Eiweiss nöthig hat. Diese Voraussetzung lässt sich allerdings nach den bisherigen Untersuchungen mit Sicherheit nicht beantworten. Doch ist zu beachten, dass man den gewöhnlichen bedeutenden Eiweissbedarf auch erklären kann, ohne dass man dies auf ein absolutes Bedürfniss des Körpers an Eiweiss beziehen muss.

Der erste Punkt ist, dass die eiweissreichen Nahrungsmittel nicht nur mit zu den gebräuchlichsten zählen, sondern wohl auch die besten Nahrungsmittel sind. So nimmt das fast nur eiweisshaltige Fleisch den ersten Platz nicht allein unter den Nahrungsmitteln, sondern auch unter den Genussmitteln ein. Eine wie grosse Wichtigkeit allein letzterem Umstande zuzuschreiben ist lehrt die Erfahrung, dass der Fleischbrïhe einst ein sehr hoher Nährwerth zugeschrieben wurde, während man sie jetzt nur als ein angenehmes Genussmittel betrachtet. Die Fleischnahrung bat 
aber neben den angenehmen Geschmack noch verschiedene Vorziuge als Nahrungsmittel. Die grosse Schnelligkeit, mit der es im Munde zerkleinert wird, im Magen von den Verdauungssäften angegriffen und resorbirt wird. Sein Hauptwerth liegt aber wohl darin, dass in dem Zustande in dem es gewöhnlich genossen wird, gebraten oder gesotten, bei relativ geringem Gewicht auch ein sehr geringes Volumen einnimmt. Wie $\mathrm{V}$ oit ${ }^{1}$ ) berechnete, wiegen $100 \mathrm{gr}$ gesotten $56,7 \mathrm{gr}$. Der Wassergehalt des Fleisches, der frïher $75,9 \%$ betrug, ist jetzt auf $44,3 \%$ gesunken. $300 \mathrm{gr}$ Fleisch mit $40 \mathrm{gr}$ Fett gebraten lieferten mir eine ca. $200 \mathrm{gr}$ schwere Speise, annähernd also so schwer als drei 3 Pfennig Semmeln von je $70 \mathrm{gr}$ Gewicht, aber von bedeutend geringerem Volumen (ca. den dritten Theil) als diese, obgleich das Brod noch vor den andern an Koblehydraten reichen Nahrungsmitteln, z. B. den Kartoffeln, den Vorzug des relativ geringsten Gewichtes und Volumens hat. Dabei enthielt der Braten ea. $64 \mathrm{gr}$ Eiweiss und ca. $40 \mathrm{gr}$ Fett. Es werden demnach mit dem Fleisch beträchtliche Mengen Fett, besonders wenn das Schlachtthier selbst fettreich ist und als Beigabe ferner noch Brod und an Kohlehydraten reiche Gemüse genossen. Der Vorzug des Fleisches würde also darin beruhen, dass in ihm nicht so grosse Mengen Kohlehydrate wie in dem Brod und den andern Vegetabilien enthalten sind. Da wir diese Nahrungsmittel überdies schon in ziemlicher Menge meist zu uns nehmen, bildet ein von ihnen durch den gänzlichen Mangel an Kohlehydraten so verschiedenes Nahrungsmittel eine angenehme Ergänzung und Abwechselung.

Wir greifen daher bei einer hohen Aussentemperatur, wenn wir durch Vermeidung von unnöthigen Muskelbewegungen unsere Wärmeproduktion so viel als möglich verringern und in Folge dessen weniger Nahrung nöthig haben, nicht zu den an Kohlehydraten reichen Nahrungsmitteln wie den Reis, Kartoffeln, obgleich sich dieselben gerade als Nahrungsmittel für heisse Klimate eignen sollen ${ }^{2}$ ). Vielmehr geschieht es dann wohl meist, dass wir die Kohlehydrate und besonders die Fette vermeiden und lieber Fleisch geniessen. Gerade dies könnte dafür sprechen, dass wir grössere Mengen Eiweiss absolut nothwendig haben, aber hier-

1) Vo it, Handbuch des Stoffwechsels S. 444.

2) Munk v. Uffelmann, Handbuch der Diätetik S. 375. 
gegen lässt sich der Einwand machen, dass in heissen Ländern, wo meist weniger Nahrung verzehrt wird, nicht demgemäss in der täglichen Kost das Eiweiss im Verhältniss zu den andern Nährstoffen vermehrt ist. In den meisten Fällen werden im Gegentheil eiweissarme und viel zuckerhaltige Nahrungsmittel, so vor allem der Reis genossen, während Eiweiss und Fett in einem viel geringeren Verhältniss als beim Bewohner kalter Regionen vorherrschend sind. Sie milssen dabei doch bedeutende Mengen Nahrung zu sich nehmen. Erstens um, aus den Kohlehydraten und der geringen Eiweissmenge die nöthige Wärme zu entwickeln. So sind z. B. $600 \mathrm{gr}$ Reis nöthig, um die gewöhnlichen Resorptionsverhältnisse gerechnet, die 2000 Wärmecalerien zu liefern, welche für den Menschen mindestens erforderlich sind. Zweitens müssten sie aber kolossale Mengen Reis geniessen, wenn sie dem Körper ca. $100 \mathrm{gr}$ Eiweiss zukommen lassen wollten. Dazu würden sie täglich ca. $1600 \mathrm{gr}$ Reis brauchen, was bei der Zubereitung mit möglichst wenig Wasser doch noch eine Speise von ca. $5 \mathrm{~kg} \mathrm{Ge}$ wicht und dementsprechenden Volumen ausmachen würde. Hierbei -müssten sie aber einen grossen Wärmeüberschuss entwickeln, da sie bei dieser Nahrung ca. 5000 Wärmecalorien produciren wiirden.

Weiterhin scheint die Thatsache sehr wichtig, dass man so häufig bei Personen um so mehr $\mathrm{N}$ im Urin findet, je mehr Arbeit sie leisten. Dass hierfür die Erklärung nicht genẗgt, dass der muskelreiche Körper stark Arbeitender mehr Eiweiss braucht wie der muskelschwache, ist schon oben erwähnt worden. Auch kann man in der That dieses Verhältniss anf andere Weise deuten.

Die Arbeiter leben meist von vegetabilischen d. i. an Koblehydraten reichen Nahrungsmitteln als den billigsten. In fast allen Vegetabilien, so besonders dem Brod und den Kartoffeln sind aber gewisse Mengen Eiweiss. Nimmt man nun an, dass ein Arbeiter sehr kräftig arbeitet, so wird seine Wärmeproduktion gesteigert, sein Nahrungsbedurfniss erhöht. Dies könnte er erstens dadurch befriedigen, dass er mehr von seiner gewohnten Nahrung isst.

Schon dieser Umstand würde, da er hierbei mehr Eiweiss geniesst, seinen Eiweissumsatz steigern. Besonders wird dies der Fall sein, wenn er sich von den nicht zu voluminösen Nahrungsmitteln, dem Brod, Hirse, Erbsen, Bohnen u. s. w. nährt und dabei noch viel Fett geniesst. Anders wird 
dies jedoch, wenn z. B. die Kartoffel sein Hauptnahrungsmittel ist. Hiervon nimmt er schon überdies bedeutende Mengen zu sich; um das vermehrte Nahrungsbedürfniss damit zu decken, müsste er dieses Quantum aber kolossal vermehren. Er muss deshalb an andere Stoffe denken. Entweder greift er daber zu seinem Unglück häufig zum Alcohol und zwar zu dem billigsten Getränk, dem stark alcoholischen fuselhaltigen Branntwein, der ihm die nöthige Wärme liefert, oder zu den Fetten und Eiweisskörpern. Dass letztere besonders ihm ein nahrhaftes und wenig voluminöses Nahrungsmittel liefern, ist schon oben betont worden. Wie sehr dabei die Arbeiter einen Hauptwerth auf die stark Wärme bildenden Stoffe besonders das Fett legen, erkennt man schon daraus, dass sie das fettreiche Schweinefleisch und die fetthaltigen Würste oder den Speck vor dem magern Rindfleiseh bevorzugen. Es sind also zwei Factoren für den reichlichen Eiweissgehalt der Nabrung stark arbeitender Personen wesentlich maassgebend. Zuerst das vermehrte Nahrungsbedürfniss, wobei, wie alle Nährstoffe, auch das Eiweiss vermehrt sein wird.

Zweitens die Unmöglichkeit, die bei der Arbeit sich entwickelnde Wärme allein von den meist zu voluminösen Kohlenhydraten zu bestreiten.

Zum Schluss möchte Verfasser folgende Sätze als das Ergebniss seiner Arbeit zusammenfassen:

1. Es ist möglich, dass ein kräftiger Mann erst 15 Tage, dann 10 Tage sich mit einer Kost im N-Gleichgewicht hält, deren Gehalt an N-haltigen Stoffen 5-8 gr war. Da jedoch nicht die gesammte $\mathrm{N}$-Menge als Eiweiss in den Nahrungsmitteln vorhanden war, so darf man annehmen, dass dem Organismus meist nur $30-35 \mathrm{gr}$ Eiweiss den Tag geboten wurden. Nimmt man noch. die Tage aus, an denen sehr wenig $\mathrm{N}-5$ gr oder noch geringere Mengen in 24 Stunden - eingefuhrt wurden, so kann man doch mit Sicherheit den Schluss ziehn, dass mit ca. $40-45$ gr $\mathrm{N}$-haltigen Stoffen oder 35-40 gr Eiweiss ein Gleichgewicht erreicht worden ist.

$\mathrm{Ob}$ dies noch länger gelingt, lässt sich bis jetzt noch nicht entscheiden, eben so wenig wie hoch der Bedarf des Organismus an Eiweiss eigentlich ist.

2. Es lassen sich nach unseren bisherigen Kenntnissen weder durch Versuche noch durch theoretische Erwägungen Beweise dafür beibringen, dass die körperliche Leistungsfähigkeit wesentlich 
davon abhängt, dass ziemlich bedeutende Mengen Eiweiss (durchschnittlich täglich $100-120 \mathrm{gr}$ vom erwachsenen Mann) genossen werden. Auch ist keine Gefabr, dass bei etwas geringerer Eiweissnahrung das Organeiweiss abschmilzt und ausgeschieden wird. Eine wesentliche Vermehrung des Organeiweisses findet, wenn man von der durch vergrösserte Thätigkeit bedingten compensatorischen Hypertrophie absieht, nur durch Mästung statt, d. h. unter $\mathrm{Zu}$ nahme des Fettreichthums des Organismus.

3. Die eiweissreichen Nahrungsmittel, besonders das Fleisch sind vorzügliche Genuss- und Nahrungsmittel, hauptsächlich weil sie ein ausserordentlich geringes Gewicht und Volumen haben und dann im Körper leicht zersetzlich sind. Schon aus diesen Grunde mïssen sie bei kräftig Arbeitenden neben der reichlich Koblehydrate haltenden Nahrung immer in einer gewissen Menge genossen werden. Doch ist es wohl möglich, dass sie dabei theilweise bei guter Verdaung durch Fette und auch durch nicht zu voluminöse Kohlehydrat-reiche Nahrungsmittel, die immer etwas Eiweiss enthalten, ersetzt werden, wenn dabei auch nicht die Menge der Eiweisskörper so stark wie in meinen Versuchen herabgesetzt wird. Bei der Wichtigkeit der Frage ist es aber natiirlich nothwendig, dass hierüher noch ausgedehnte Untersuchungen angestellt werden. Dass zu diesem Ersatze die von mir benutzten Speisen sich besonders eignen, soll durchaus nicht behauptet werden, vielmehr waren bei ihrer Wabl und ihrer Zubereitung so viel andere Rücksichten wie erwähnt maassgebend, dass viele andere Punkte, auf die man sonst bei der Wahl einer Kost Werth legt, so auf guten Geschmack - hier vernachlässigt werden mussten. Die Störungen, welche bei Individuen eintreten sollen, die wenig Eiweiss geniessen, meistens aus dem Grunde, dass sie die theueren eiweissreichen Nahrungsmittel nicht bezahlen können, lassen sich vielleicht eher auf schlecht gewählte und schlecht zubereitete Nahrung sowie andere hygienische Missstände zurückführen, als auf den Mangel an Eiweiss: 